\title{
Erratum: (Modified) Fredholm Determinants for Operators with Matrix-Valued Semi-Separable Integral Kernels Revisited
}

Fritz Gesztesy and Konstantin A. Makarov

Integr. equ. oper. theory 47 (2003) 457-497

Due to an unfortunate technical problem, all $L^{1}$ and $L^{2}$-spaces are displayed as $L 1$ and $L 2$ throughout this paper.

In addition, the following related typographical errors occurred:

p. 469: In lines $13,15,20$, and 22, replace $\alpha 2$ by $\alpha^{2}$.

p. 473: In line 8, replace $P 2$ by $P^{2}$.

In lines 8 and 13 , replace $-d 2 / d x 2$ by $-d^{2} / d x^{2}$.

p. 480: In (4.78), replace $\operatorname{det}_{\mathbb{C} 2}$ by $\operatorname{det}_{\mathbb{C}^{2}}$.

p. 481: In (4.88), replace $\mathbb{R} 2$ by $\mathbb{R}^{2}$.

p. 482 : In line 6 , replace $L 2(\mathbb{R} ; d x) 2$ by $L^{2}(\mathbb{R} ; d x)^{2}$.

p. 489: The following assumption should be added right after equation (5.18): "For the remainder of this section we always assume that $\zeta_{n} \neq \zeta_{n^{\prime}}$ for $n \neq n^{\prime \prime \prime}$. This applies, in particular, to Theorem 5.3. (We thank A. Böttcher for this comment.)

It has been brought to our attention that what we called "the analog of Day's formula for Wiener-Hopf operators," more appropriately, should be called Böttcher's formula as it was first published in 1989 by A. Böttcher in [1].

The electronic-only corrected version of our paper (with all these typos removed) has the following DOI: 10.1007/s00020-003-1279-z. It will appear on SpringerLink at the very end of issue 4 of Vol. 48 (2004). 
Fritz Gesztesy and Konstantin A. Makarov

Department of Mathematics, University of Missouri, Columbia, MO 65211, USA

e-mail: fritz@math.missouri.edu

URL: http://www.math.missouri.edu/people/fgesztesy.html

e-mail: makarov@math.missouri.edu

URL: http://www .math.missouri.edu/people/kmakarov.html

Submitted: December 18, 2003

(1D) To access this journal online:

(40) http://www.birkhauser.ch 